\title{
Sorafenib after Combination Therapy with Gemcitabine plus Doxorubicine in Patients with Sarcomatoid Renal Cell Carcinoma: A Prospective Evaluation
}

\author{
M. Staehler, N. Haseke, A. Roosen, T. Stadler, M. Bader, M. Siebels, A. Karl, C. G. Stief \\ Department of Urology, University of Munich, Klinikum Grosshadern, Munich, Germany
}

\begin{abstract}
Background: Sarcomatoid renal cell cancer (RCC) is a distinct histological variant of RCC that is associated with rapid progression and a poor prognosis. The optimal treatment for patients with sarcomatoid RCC remains to be defined. Gemcitabine plus doxorubicine (GD) has shown some efficacy, however durability of response is limited. We carried out a prospective, open-label study to investigate the efficacy and safety of sorafenib in patients after GD failure in sarcomatoid RCC.

Methods: Fifteen patients with pure sarcomatoid RCC and objective progressive disease were treated with GD (gemcitabine $1500 \mathrm{mg} / \mathrm{m}^{2}$, doxorubicine 50 $\mathrm{mg} / \mathrm{m}^{2}$ administered by weekly intravenous infusion) until progression of disease. Subsequently 9 patients were switched to sorafenib (400 mg twice daily). Tumor response was measured by physical examination and computerized tomography scans and evaluated according to Response Evaluation Criteria in Solid Tumors criteria.
\end{abstract}

Results: Median time to progression (T'TP) under GD was 6.6 months (range $0.8-8$ months). During GD treatment there were no remissions and 6 patients died from progressive disease. Median TTP for the $9 \mathrm{pa}-$ tients switched to sorafenib was 10.9 months (range $0.6-25.5$ months). During sorafenib therapy one patient had a partial remission lasting for 3 months and 4 patients experienced stable disease with a duration of 3 to 9 months. Four patients immediately progressed on sorafenib treatment but had a slower dynamic of tumor progression than under GD. Dosing in both treatment phases was generally well tolerated with manageable toxicities and no requirement for dose reduction.

Conclusions: Chemotherapy with GD was ineffective in our patients with pure sarcomatoid RCC. Subsequent anti-angiogenic treatment using the multi-tyrosine kinase inhibitor sorafenib resulted in additional progression-free survival in 5 of 9 patients. Further evaluation of targeted anti-angiogenic agents for the treatment of sarcomatoid RCC is warranted.

Key words: renal cell carcinoma, sarcomatoid, chemotherapy, sorafenib, multi-kinase inhibitor, survival, angiogenesis

\section{BACKGROUND}

Sarcomatoid renal cell carcinoma (RCC) is a distinct histological variant of RCC observed in around $0.7 \%$ to $13.2 \%$ of renal parenchymal carcinomas [1-4]. This form of the disease is associated with rapid progression and a very poor prognosis and there is currently no uniform consensus regarding the treatment of patients with metastatic sarcomatoid RCC [5]. Because most histological subtypes of RCC are considered to be highly resistant to cytotoxic chemotherapy [6-8], systemic therapy of RCC has, until recently, been based mainly on immunotherapy with interferon (IFN) and or interlekin-2 (IL-2). However, response rates are consistently low and of limited durability [9]. With sarcomatoid RCC, the very fast and aggressive growth of the tumor provides more of a rationale for the use of chemotherapy in patients with this sub-type of the disease. Several approaches to the treatment of sarcomatoid RCC with chemotherapeutic agents have been investigated with varying degrees of success. Regimes based on the combination of gemcitabine, docetaxel and carboplatin have shown some effect in individual cases $[10,11]$. A number of case studies and sub-analyses have reported complete responses in patients treated with combinations including doxorubicin [12-14], although one larger study, in 23 patients, reported the combination of ifosfamide and doxorubicin to be ineffective [15]. In contrast, Nanus et al. confirmed the effectiveness of doxorubicine in 18 patients with rapidly growing metastatic RCC or sarcomatoid RCC treated with doxorubicine and gemcitabine [16]. With this regimen a complete response was observed in two patients, a partial response in five patients, a mixed response in three patients and stable disease in one patient. The median duration of response was 5 months.

The introduction of multi-kinase inhibitors targeting the VEGF-R and PDGF-R pathway, such as sorafenib and sunitinib, has transformed the treatment of metastatic or advanced RCC $[17,18]$. In patients previously treated with immunotherapy sorafenib doubled progression free survival from 12 to 24 weeks with a manageable toxicity profile [17]. It is not yet know whether these new inhibitors will show similar activity in patients with sarcomatoid RCC. Unfortunately, data from the phase III clinical trials of target- 
ed agents cannot be applied to this question since the inclusion criteria specified the presence of predominantly clear cell carcinoma, thus excluding patients with pure sarcomatoid RCC.

We carried out a prospective, open-label study to investigate the efficacy and safety of sorafenib in patients with metastatic sarcomatoid RCC who progress on prior chemotherapy. Based on the encouraging results of Nanus et al. [16], at the time of initiation of the study $(01 / 2007)$ the standard treatment for all patients with metastatic sarcomatoid RCC in our clinic was the combination of gemcitabine and doxorubicine (GD). Although multi-kinase inhibitors were yet to be approved for RCC treatment we commenced the study in anticipation of the imminent availability of sorafenib.

\section{PATIENTS AND METHODS}

Study participants were treatment-naïve patients with metastatic RCC confirmed by high resolution computerized tomography (CT) scans. All patients had previous nephrectomy, pure sarcomatoid RCC, based on advanced immunhohistochemical evaluation by a single pathologist, and progressive disease determined by imaging over at least one month. Patients had to have an Eastern Cooperative Oncology group (ECOG) performance status of 0 or 1 , normal cardiac and organic function, assessed by electrocardiography (ECG), and normal serum analysis was mandatory. Patients with brain metastases, evidence of New York Heart Association functional Class III or IV heart disease or severe arrhythmias (including first, second or third degree heart block) were excluded from the observational study. The risks and benefits of the therapy were thoroughly discussed in detail with each patient before treatment initiation. Since the study was a non-interventional study, no formal ethics committee and competent authority approvals were required and no patient informed consent had to be obtained.

This was a single centre, non-randomized, prospective, open-label non-interventional evaluation of treatment with gemcitabine and doxorubicine followed by sorafenib in patients with disease progression. The subjects received therapy according to medical need and the recommendations provided in the summary of product characteristics Patients received doxorubicin as intravenous (i.v.) bolus infusion plus gemcitabine by i.v. infusion over 1 hour. The initial doses were $1500 \mathrm{mg} / \mathrm{m}^{2}$ for gemcitabine and $50 \mathrm{mg} / \mathrm{m}^{2}$ for doxorubicin. Recombinant human granulocyte-colonystimulating factor was administered at $5 \mu \mathrm{g} / \mathrm{kg}$ per day by subcutaneous injection if necessary. The duration of one cycle was 4 weeks with chemotherapy administered every week. Gemcitabine dose reductions of 20$30 \%$ were planned in the event of toxicity. Treatment with gemcitabine plus doxorubicin was continued until disease progression occurred. In patients who progressed on gemcitabine plus doxorubicin, sorafenib was initiated at a dose of $400 \mathrm{mg}$ orally, twice daily. For toxicity management sorafenib dose could be reduced by $200 \mathrm{mg} /$ day until side effects diminished and then re-escalated again.

Sorafenib treatment was continued until confirmed disease progression was observed.
Pre-treatment evaluation included a complete medical history with ECOG performance status and physical examination. Baseline analyses included ECG, CT scans of the brain, thorax and whole abdomen, complete blood count and measurement of serum levels of creatinine, aspartate aminotransferase, alanine aminotransferase, gamma-GT, lactate-dehydrogenase activity, calcium, albumin and thyroid-stimulating hormone. Bone scans were performed if there was clinical evidence of metastases of the arms or legs. On-treatment evaluation was performed every 8 weeks ( \pm 3 days) throughout the study. Tumor response was evaluated by physical examination and CT-scans of the thorax and abdomen. CT scans were evaluated according to the according to Response Evaluation Criteria in Solid Tumors (RECIST) criteria [19]. A complete response (CR) was defined as the disappearance of all lesions in clinical and radiological examinations. Partial response was defined as a $30 \%$ decrease of the summed diameters of no more than ten extra-osseous lesions without any lesions progressing. Stable disease was an increase in no more than $20 \%$ of the summed diameters compared to the smallest achieved size or a reduction of the summed diameters of less than $30 \%$. Progressive disease was an increase in the sum of the lesions of more than $20 \%$ compared to the summed smallest diameters achieved at any time or the appearance of any new lesions. Response had to be confirmed after 6 weeks by another tumor measurement. Toxicity was graded according to the National Cancer Institute Common toxicity Criteria, Version 3.0.

\section{RESULTS}

Fifteen patients were treated, 4 female and 11 male. Patient characteristics are given in Table 1 . The median age was 58 years (range 39-80 years). All patients had undergone nephrectomy and had histologicaly confirmed pure sarcomatoid differentiation of RCC. Clear-cell or other underlying histological entities of RCC were not identified in any patients. All patients had 2 or more metastatic sites and were classified as low risk according to the Memorial Sloan Kettering Cancer Center 1999 risk assessment score.

Patients received a median of 4 courses per patient (range 1-7 courses) of gemcitabine plus doxorubicin. Chemotherapy was generally well tolerated with no Grade 3 or 4 toxicities. Diarrhea and hypertension were the most common side effects. No patients required dose attenuation. None of the patients experienced remission with gemcitabine plus doxorubicin. The median time to progression (T'TP) under gemcitabine plus doxorubicin was 6.6 months (range $0.8-8$ months). Six patients died from progressive disease prior to being switched to sorafenib.

Nine patients were treated with sorafenib. One patient had a partial remission (Fig. 1) lasting for 3 months and four patients experienced stable disease with a duration of 3 to 9 months. The four remaining patients continued to experience progressive disease under sorafenib, however, progression was slower than that observed under gemcitabine plus doxorubicin as evidenced by smaller increases in tumor size between measurements. The median TTP on sorafenib treat- 
Table 1. Patient characteristics.

\begin{tabular}{lcc}
\hline & Baseline & $\begin{array}{c}\text { At time of switch } \\
\text { to sorafenib }\end{array}$ \\
\hline $\begin{array}{l}\text { Number of patients } \\
\text { Median age (range) in yrs }\end{array}$ & 15 & 9 \\
Gender, $\mathrm{n}$ & $(39-80)$ & \\
$\quad$ Male & 11 \\
Female & 4 \\
ECOG Performance status, n & \\
$0-1$ & 7 \\
2 & 4 \\
3 & 3 \\
4 & 0 \\
Previous nephrectomy, $\mathrm{n}$ & 14 \\
No. of disease sites, $\mathrm{n}$ & \\
1 & \\
2 & 0 \\
$>=3$ & 10 \\
Metastatic sites, $\mathrm{n}$ & 45 \\
Lung & \\
Lymph nodes & \\
Bone & \\
Peritoneum & 3 \\
Pancreas & 4 \\
Liver & \\
CNS & 4 \\
\hline
\end{tabular}

GD, gemcitabine plus doxorubicin ECOG, Eastern Cooperative Oncology Croup; CNS, central nervous system

Table 2. Initial stage and best response with sorafenib.

\begin{tabular}{lcc}
\hline TNM stage & $\begin{array}{c}\text { Best response } \\
\text { on GD }\end{array}$ & $\begin{array}{c}\text { Best response } \\
\text { on sorafenib }\end{array}$ \\
\hline pT2 pN0 & SD & PR \\
pT4 pNx cM1 & SD & SD \\
pT3b pN0 cM0 & SD & SD \\
pT1b Nx pM1 R2 & SD & SD \\
pT3a pN0 cM0 & SD & SD \\
pT2 pN0 cM0 & SD & PD \\
pT3a Nx R1 & SD & PD \\
pT3a pN2 pM1 & SD & PD \\
pT4 Nx cMx & SD & PD \\
pT3b pN0 pM1 & SD & - \\
pT1b Nx cM0 & PD & - \\
pT3b pN0 cM0 & PD & - \\
pT3b pN1 cM1 & PD & - \\
pT2 pN0 cM1 & PD & - \\
\hline
\end{tabular}

ment was 10.9 months (range 0.6 - 25.5 months). Among the nine patients treated with sorafenib overall survival (OS) from initial diagnosis was 36.4 months and OS from diagnosis of metastatic disease was 8.0 months.
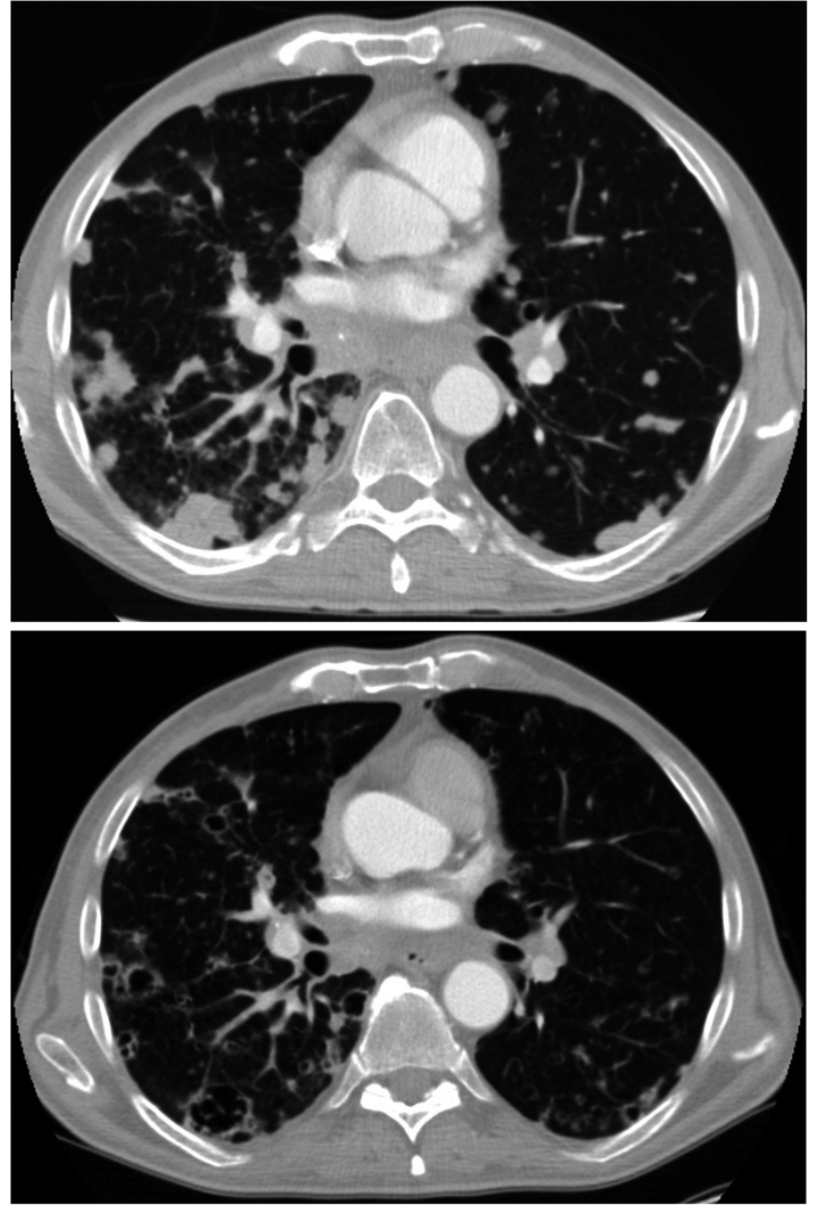

Fig. 1. CT scans for a patient of a patient with bilateral disseminated pulmonary metastasesshowing a partial response on sorafenib treatment; a) solid lesions pretreatment, b) partial response after 4 months of sorafenib treatment with cavernisation and shrinkage of the lesions.

\section{DiscusSION}

The treatment of non-clear cell histological variants of RCC with chemotherapy or immunotherapy is a subject of controversy. Chemotherapy has clearly been proven to be ineffective for the treatment of clear-cell, metastatic RCC [6, 7, 20-23] In contrast, several small studies and case reports indicate that chemotherapeutic combinations can be beneficial in aggressive subtypes of RCC, such as collecting duct carcinomas, sarcomatoid RCC and rapidly progressing clear cell RCC $[4,12,13,24,25]$. Although these reports are encouraging, contradictory data has also been published [15]. No clear conclusions can be drawn since the definitions of response vary from study to study and are not consistent with RECIST criteria. Variations in response criteria have been shown to significantly alter estimates of time to progression [26] and this may account, to some extent, for the lack of consistency between reported TTP data.

Nanus et al. previously reported successful GC treatment of RCC patients with sarcomatoid or rapidly progressing tumors. Response rates in this study were higher than observed by us, with $7 / 18$ patients experiencing a partial or complete response. However, 
the median TTP was similar (5 months [range, 2-21 months]). Of note, only 14 of these patients were nephrectomized and the percent of sarcomoid tumors in each specimen was not measured. In contrast, in our study all patients were nephrectomized and showed histological evidence of pure sarcomatoid RCC with no clear-cell or other underlying histological entities of RCC identified. In addition, our study used more accurate response evaluation criteria based on high resolution CT scans in a very strict time-frame. This might contribute to our apparently very low response rate with GD.

The introduction of tyrosine-kinase inhibitors has radically changed the treatment of advanced clear-cell RCC. Although patients with non-clear-cell RCC were not included in the phase III clinical trials, sub-analyses of expanded access programs along with some small clinical studies do indicate that sorafenib may have some activity in these patients, including those with sarcomatoid features [27-30]. In our study sorafenib treatment was able to stabilize the disease in the majority of the patients (5/9) who had progressed on previous GD therapy. That only one patient experienced a PR is consistent with the $10 \%$ partial response rate published for treatment with sorafenib after prior cytokine therapy in clear-cell RCC [17]. Sorafenib has been show to cause interior tumour necrosis rather than tumour shrinkage [31]. Since the RECIST criteria are based only on reductions in exterior tumour size response rates may not provide a clear picture of the actual clinical benefit of sorafenib

Our data are the first prospective data on the use of a tyrosine kinase inhibitor in a well defined and rare entity of RCC other than clear-cell histology. Although little is known about sarcomatoid RCC and alterations of its angiogenic pathways, accumulating clinical evidence makes it hopeful that we may see this mechanisms of action leading to results that are comparable with those seen in the first- and secondline trials in patients with clear-cell histology $[17,18$, 32]. Perhaps future regimes combining chemotherapy and anti-angiogenic drugs could aid in the struggle against extreme aggressive RCC entities that were resistant to chemotherapy and immunotherapy in the past.

\section{CONCLUSiOnS}

Chemotherapy is of limited efficacy for sarcomatoid RCC, as seen for other RCC histologies [6, 10]. Chemotherapy with GD was ineffective in our patients with pure sarcomatoid RCC. Subsequent anti-angiogenic treatment using the multi-tyrosine kinase inhibitor sorafenib resulted in additional progressionfree survival in 5 of 9 patients. Based on the results of this small, single centre, prospective analysis further evaluation of targeted anti-angiogenic agents for the treatment of sarcomatoid RCC in a larger multicentre clinical trial is warranted.

Competing interests: Michael Staehler received honoraria from Bayer, Pfizer, Novartis, Wyeth and Roche. All other authors have no competing interests declared.

\section{REFERENCES}

1. Bannowsky A, Leuschner I, Schiller H, Bothe K, Osmonov D, Junemann KP et al. [Sarcomatoid renal cell carcinoma. A rare and aggressive variation of primary renal cell carcinoma]. Urologe A 2007: 46(4):406-411.

2. Pantuck AJ, Zisman A, Dorey F, Chao DH, Han KR, Said J et al. Renal cell carcinoma with retroperitoneal lymph nodes. Impact on survival and benefits of immunotherapy. Cancer 2003: 97(12):2995-3002.

3. Stadler WM, Halabi S, Rini B, Ernstoff MS, Davila E, Picus $\mathrm{J}$ et al. A phase II study of gemcitabine and capecitabine in metastatic renal cancer: a report of Cancer and Leukemia Group B protocol 90008. Cancer 2006: 107(6):1273-1279.

4. Cangiano T, Liao J, Naitoh J, Dorey F, Figlin R, Belldegrun A. Sarcomatoid renal cell carcinoma: biologic behavior, prognosis, and response to combined surgical resection and immunotherapy. J Clin Oncol 1999: 17(2):523528.

5. National Comprehensive Cancer network. NCCN clinical practice guidelines in oncology: kidney cancer. Available at www nccn org: accessed 28 Aug 2008.

6. Amato RJ. Chemotherapy for renal cell carcinoma. Semin Oncol 2000: 27(2):177-186.

7. Yagoda A, bi-Rached B, Petrylak D. Chemotherapy for advanced renal-cell carcinoma: 1983-1993. Semin Oncol 1995: 22(1):42-60.

8. Ljungberg B, Hanbury DC, Kuczyk MA, Merseburger AS, Mulders PF, Patard JJ et al. Guidelines on renal cell carcinoma. Eur Urol 2007: 51(6):1502-1510.

9. Bleumer I, Oosterwijk E, De MP, Mulders PF. Immunotherapy for renal cell carcinoma. Eur Urol 2003: 44(1):65-75.

10. David KA, Milowsky MI, Nanus DM. Chemotherapy for non-clear-cell renal cell carcinoma. Clin Genitourin Cancer 2006: 4(4):263-268.

11. Hoshi S, Satoh M, Ohyama C, Hiramatu M, Watanabe R, Hagisawa $\mathrm{S}$ et al. Active chemotherapy for bone metastasis in sarcomatoid renal cell carcinoma. Int J Clin Oncol 2003: 8(2):113-117.

12. Bangalore N, Bhargava P, Hawkins MJ, Bhargava P. Sustained response of sarcomatoid renal-cell carcinoma to MAID chemotherapy: case report and review of the literature. Ann Oncol 2001: 12(2):271-274.

13. Culine S, Bekradda M, Terrier-Lacombe MJ, Droz JP. Treatment of sarcomatoid renal cell carcinoma: is there a role for chemotherapy? Eur Urol 1995: 27(2):138-141.

14. Lupera H, Theodore C, Ghosn M, Court BH, Wibault P, Droz JP. Phase II trial of combination chemotherapy with dacarbazine, cyclophosphamide, cisplatin, doxorubicin, and vindesine (DECAV) in advanced renal cell cancer. Urology 1989: 34(5):281-283.

15. Escudier B, Droz JP, Rolland F, Terrier-Lacombe MJ, Gravis G, Beuzeboc P et al. Doxorubicin and ifosfamide in patients with metastatic sarcomatoid renal cell carcinoma: a phase II study of the Genitourinary Group of the French Federation of Cancer Centers. J Urol 2002: 168(3):959-961.

16. Nanus DM, Garino A, Milowsky MI, Larkin M, Dutcher JP. Active chemotherapy for sarcomatoid and rapidly progressing renal cell carcinoma. Cancer 2004: 101(7): 1545-1551.

17. Escudier B, Eisen T, Stadler WM, Szczylik C, Oudard S, Siebels $\mathrm{M}$ et al. Sorafenib in advanced clear-cell renal-cell carcinoma. N Engl J Med 2007: 356(2):125-134.

18. Motzer RJ, Hutson TE, Tomczak P, Michaelson MD, Bukowski RM, Rixe $\mathrm{O}$ et al. Sunitinib versus interferon alfa in metastatic renal-cell carcinoma. N Engl J Med 2007: 356(2):115-124. 
19. Therasse P, Arbuck SG, Eisenhauer EA, Wanders J, Kaplan RS, Rubinstein L et al. New guidelines to evaluate the response to treatment in solid tumors. European Organization for Research and Treatment of Cancer, National Cancer Institute of the United States, National Cancer Institute of Canada. J Natl Cancer Inst 2000: 92(3):205-216.

.20 Bruntsch U, Heinrich B, Kaye SB, de Mulder PH, van Oosterom A., Paridaens $\mathrm{R}$ et al. Docetaxel (Taxotere) in advanced renal cell cancer. A phase II trial of the EORTC Early Clinical Trials Group. Eur J Cancer 1994: 30A(8):1064-1067.

21. Fizazi K, Rolland F, Chevreau C, Droz JP, Mery-Mignard $\mathrm{D}$, Culine $\mathrm{S}$ et al. A phase II study of irinotecan in patients with advanced renal cell carcinoma. Cancer 2003: 98(1):61-65.

22. Rini BI, Vogelzang NJ, Dumas MC, Wade JL, III, Taber DA, Stadler WM. Phase II trial of weekly intravenous gemcitabine with continuous infusion fluorouracil in $\mathrm{pa}-$ tients with metastatic renal cell cancer. J Clin Oncol 2000: 18(12):2419-2426.

23. Yagoda A, Petrylak D, Thompson S. Cytotoxic chemotherapy for advanced renal cell carcinoma. Urol Clin North Am 1993: 20(2):303-321.

24. Gollob JA, Upton MP, DeWolf WC, Atkins MB. Longterm remission in a patient with metastatic collecting duct carcinoma treated with taxol/carboplatin and surgery. Urology 2001: 58(6):1058.

25. Milowsky MI, Rosmarin A, Tickoo SK, Papanicolaou N, Nanus DM. Active chemotherapy for collecting duct carcinoma of the kidney: a case report and review of the literature. Cancer 2002: 94(1):111-116.

26. Schwartz LH, Mazumdar M, Wang L, Smith A, Marion S, Panicek DM et al. Response assessment classification in patients with advanced renal cell carcinoma treated on clinical trials. Cancer 2003: 98(8):1611-1619.

27. Beck J, Bajetta E, Escudier B, Negrier S, Keilholz U, Szczylik C et al. A large open-label, non-comparative, phase III study of the multi-targeted kinase inhibitor sorafenib in European patients with advanced renal cell carcinoma. Eur J Cancer Suppl 2007: 5(4):300-abstract No.\#4506.
28. Choueiri TK, Plantade A, Elson P, Negrier S, Ravaud A, Oudard $S$ et al. Efficacy of sunitinib and sorafenib in metastatic papillary and chromophobe renal cell carcinoma. J Clin Oncol 2008: 26(1):127-131.

29. Procopio G, Verzoni E, Gevorgyan A, Mancin M, Pusceddu S, Catena L et al. Safety and activity of sorafenib in different histotypes of advanced renal cell carcinoma. Oncology 2007: 73(3-4):204-209.

30. Golshayan AR, George S, Heng DY, Elson P, Wood LS, Mekhail TM et al. Metastatic sarcomatoid renal cell carcinoma treated with vascular endothelial growth factor-targeted therapy. J Clin Oncol 2009: 27(2):235-241.

31. Abou-Alfa GK, Schwartz L, Ricci S, Amadori D, Santoro A, Figer A et al. Phase II study of sorafenib in patients with advanced hepatocellular carcinoma. J Clin Oncol 2006: 24(26):4293-4300.

32. Hudes G, Carducci M, Tomczak P, Dutcher J, Figlin R, Kapoor A et al. Temsirolimus, interferon alfa, or both for advanced renal-cell carcinoma. N Engl J Med 2007: 356(22):2271-2281.

Received: November 26, 2009 / Accepted: January 12, 2010

Address for correspondence:

Dr. Michael Staehler

Department of Urology

University of Munich

Klinikum Grosshadern

Marchioninistr. 15

81377 München

Germany

Phone: +498970953722

Fax: $\quad$ +498970956722

E-mail: michael.staehler@med.uni-muenchen.de 